\title{
Transtornos mentais comuns entre trabalhadores da zona urbana de Feira de Santana-BA, ,**
}

\author{
Common mental disorders among workers in the urban area of \\ Feira de Santana - Bahia-Brazil
}

\begin{abstract}
1 Programa de Pós-Graduação em Saúde Coletiva da Universidade Estadual de Feira de Santana, Feira de Santana, Bahia, Brasil.

2 Professora Titular do Departamento de Saúde da Universidade Estadual de Feira de Santana, Feira de Santana, Bahia, Brasil.

*Trabalho baseado na dissertação de mestrado intitulada Transtornos mentais comuns entre trabalhadores da zona urbana de Feira de Santana-BA, defendida em 2007, no Programa de Pós-Graduação em Saúde Coletiva da Universidade Estadual de Feira de Santana (UEFS).
\end{abstract}

***: Projeto financiado pela Fundação de Amparo à Pesquisa do Estado da Bahia (Fapesb) - Processo no 99104245.

\section{Contato:}

Mônica Dantas Farias

Rua Manoel Alves Pereira, 381 - casa

10 - Bairro Capuchinhos

Feira de Santana-Bahia

CEP: 44076-100

E-mail:

monicadantasfarias@yahoo.com.br

Recebido: 23/01/2010

Revisado: $13 / 10 / 2010$

Aprovado: 21/10/2010

\section{Resumo}

Objetivo: Estimar a prevalência de transtornos mentais comuns (TMCs) em trabalhadores de Feira de Santana/BA, avaliando a ocorrência desses transtornos segundo diferentes aspectos da vida: características sociodemográficas, do trabalho profissional (incluindo aspectos psicossociais) e atividades de lazer. Métodos: Estudo de corte transversal, de caráter exploratório, com amostra aleatória de trabalhadores de Feira de Santana. Um questionário estruturado com questões sobre características sociodemográficas, trabalho profissional (incluindo aspectos psicossociais) e atividades de lazer foi utilizado. Os TMCs foram avaliados pelo SRQ-20 em 2002. Para análise de associação entre TMC e as variáveis de interesse, foram conduzidas análise bivariada e análise de regressão logística. Resultados: Foram estudados 1.311 trabalhadores. A prevalência global de TMC foi 25,2\%. As variáveis estatisticamente associadas à TMC, no modelo final de análise, foram: sexo feminino $(R P=3,04 ; 2,34-3,95)$, baixa escolaridade $(R P=2,02 ; 1,57-2,60)$, renda mensal baixa $(\mathrm{RP}=1,78 ; 1,12-2,84)$, ausência de atividades de lazer $(\mathrm{RP}=1,35 ; 1,10$ 1,66) e trabalho de alta demanda psicológica $(\mathrm{RP}=1,45 ; 1,14-1,84)$. Conclusão: Evidenciou-se associação entre TMC e características sociodemográficas e do trabalho. Atenção especial deve ser dada a essas características para a promoção da saúde mental entre trabalhadores.

Palavras-chave: transtornos mentais comuns; trabalhadores; SRQ-20; saúde mental.

\begin{abstract}
Objective: To estimate the prevalence of common mental disorders (CMD) among workers in Feira de Santana city, Bahia, Brazil, according to sociodemographic characteristics, occupation, work psychosocial aspects, and leisure activities. Methods: Exploratory cross sectional study, with random sampling of workers of Feira de Santana. A structured questionnaire on sociodemographic characteristics, professional work, psychosocial aspects at work, and leisure activities have been used in 2002. CMD was evaluated by using the SRQ-20. Logistic regression techniques were used to analyze the association between CMD and the variables of interest. Results: 1.311 workers have been studied and the prevalence of CMD was $25.2 \%$. The characteristics statistically associated to CMD, in the final model of analysis, were: female ( $P R=3.04 ; 2.34-3.95)$, low education level ( $P R=2.02$; 1.57-2.60), low monthly income $(P R=1.78 ; 1.12-2.84)$, absence of leisure activities $(P R=1.35 ; 1.10-1.66)$, and work with high psychological demand $(P R=1.45 ; 1.14-$ 1.84). Conclusion: Association between CMD and several sociodemographic characteristics have been evidenced. Special attention must be given to these characteristics in order to promote worker's mental health.
\end{abstract}

Keywords: common mental disorders; workers; SRQ 20; mental health. 


\section{Introdução}

Estudos sobre as condições de trabalho têm demonstrado a existência de fatores de agressão à saúde relacionada às características do ambiente laboral (DEJOURS, 1992; CHECKOWAY; PEARCE; KRIEBEL, 2004; SCHNALL; DOBSON; ROSSKAM, 2009). As situações a que os trabalhadores estão submetidos - tais como sobrecarga de trabalho (acúmulo de tarefas), carga horária de trabalho extensa ou ausência de trabalho, rapidez na realização da tarefa, fadiga por esforço físico e mudanças nos processos e na organização do trabalho - podem ser fatores determinantes de diversas patologias, incluindo desde os transtornos de ajustamento ou reações ao estresse até depressões graves e incapacitantes, que podem variar de acordo com o contexto e a forma como o indivíduo responde às situações de experiências vivenciadas. Segundo Laurell e Noriega (1989), as condições de trabalho e suas patologias estão estreitamente relacionadas à organização do trabalho vigente em um determinado espaço social, sendo definidas por valores e regras da sociedade e ao mesmo tempo os definindo. No Brasil, a estrutura ocupacional formal e informal e a coexistência de realidades sociais e econômicas muito distintas, envolvendo modernidade e atraso, riqueza e pobreza, dificultam os estudos que incorporam a categoria ocupação, uma vez que um mesmo trabalhador pode exercer diferentes ocupações e estar exposto a diversas condições de trabalho ao longo da vida ou simultaneamente (no caso de múltiplos empregos) (POSSAS, 1989). As condições de vida e trabalho às quais o indivíduo está exposto desencadeiam riscos à saúde; como essas condições não são as mesmas para todos, os riscos também não se distribuem igualmente na sociedade.

Diversos estudos apontam a importância das condições socioeconômicas como fator determinante dos padrões de morbimortalidade das populações, com associação positiva entre nível socioeconômico e condição de saúde (LUDERMIR; MELLO-FILHO, 2002; BARRETO; GIATTI, 2006).

Assim, conhecer as condições e as situações de exposição e os efeitos específicos que produzem na saúde e na vida dos trabalhadores são imprescindíveis para que as ações de intervenção e as medidas de proteção à saúde possam ser adequadamente planejadas, executadas e alcancem os resultados desejados.

Aspectos relativos à saúde mental e ao trabalho têm ganhado relevo nos últimos anos. Elevadas prevalências de transtornos mentais e de registros de sofrimento psíquico nas organizações são indicadores importantes da magnitude do problema no contexto atual (LUDERMIR; MELO-FILHO, 2002; ARAÚJO; GRAÇA; 2003; BRANT et al., 2004; NASCIMENTO-SOBRINHO et al., 2006). As recentes e profundas mudanças no mundo do trabalho têm tido papel fundamental na conformação de um contexto pouco favorável à saúde dos trabalhadores.
As transformações nas formas de organizar o trabalho e o mercado impuseram importantes mudanças na vida dos trabalhadores: redução dos direitos sociais, reformas nos contratos de trabalho, supressão de postos de trabalho, aumento do subemprego ("bicos”), ameaça constante de desemprego, precarização das condições de trabalho e de vida em geral (RAMALHO, 1997).

Essas mudanças geram situações de intenso estresse para os trabalhadores: em face do aumento e da diversificação das exigências do trabalho, elevam-se os sentimentos de incapacidade em responder adequadamente a essas demandas. O estresse é resultado de uma relação particular entre a pessoa, seu ambiente e as circunstâncias às quais está exposta (FRANÇA; RODRIGUES, 2002). O estresse, por si só, não desencadeia uma disfunção ou doença, mas, a depender de como e por quanto tempo as situações geradoras de estresse se mantém, pode representar exposição e risco à saúde, contribuindo para a ocorrência de sofrimento e doenças. Assim, a vivência e a exposição contínua e em níveis elevados de demandas ambientais, associadas à falta de recursos em geral (individuais, psicológicos, ambientais, sociais), ampliam situações que podem ser nocivas aos trabalhadores. Aspectos relativos à organização do trabalho por ordenarem como o trabalho será feito, seu ritmo, a divisão de tarefas e de atribuições entre os trabalhadores e o tipo de relações entre as pessoas podem estruturar fatores de risco à saúde e à vida dos trabalhadores.

Os estudos de Dejours (1992) sobre as condições de trabalho têm demonstrado a existência de fatores de agressão à saúde relacionada ao trabalho. Ao analisar a inter-relação entre saúde mental e atividade laboral, considera a organização do trabalho como elemento estruturante dos efeitos negativos ou positivos sobre o funcionamento psíquico e a vida mental do trabalhador. Evidências empíricas nesta direção têm sido também observadas em estudos epidemiológicos, corroborando a hipótese de que situações de exposição prolongada ao estresse estão associadas a efeitos negativos sobre a saúde, destacadamente para a saúde mental (ARAÚJO et al., 2003).

Dentre os riscos ocupacionais geradores de estresse, tem-se privilegiado os fatores psicossociais.

Para análise dos fatores psicossociais em situações específicas de trabalho, um dos modelos mais utilizados tem sido o proposto por Karasek (1979), o denominado modelo demanda-controle. Este modelo, com abordagem bidimensional, considera como principais elementos estruturadores do ambiente de trabalho as demandas psicológicas (exigências psicológicas na realização das tarefas) e o controle sobre o próprio trabalho.

O controle compreende aspectos referentes ao uso de habilidades (criatividade, aprendizado de coisas novas, variedade de tarefas realizadas e desenvolvimento de habilidades especiais) e à autoridade decisória (liberdade de decidir como realizar as tarefas, pos- 
sibilidade de opinar sobre o trabalho e de influenciar a política gerencial). A demanda psicológica refere-se às exigências psicológicas que o trabalhador enfrenta na realização das suas tarefas: concentração intensa, pressão do tempo para realização de tarefas, ritmo e volume de tarefas a serem realizadas (KARASEK; THEORELL, 1990; ARAÚJO; KARASEK, 2008).

A combinação de níveis de controle e de níveis de demanda psicológica estrutura quatro tipos básicos de experiência no trabalho: baixa exigência (alto controle e baixa demanda), trabalho ativo (alto controle e alta demanda), trabalho passivo (baixo controle e baixa demanda) e alta exigência (baixo controle e alta demanda). Situações de trabalho envolvendo baixo controle sobre o próprio trabalho e elevadas demandas psicológicas, segundo o modelo de Karasek (1979), estruturam condições nocivas à saúde, podendo produzir adoecimento físico e mental.

Além dos aspectos acima mencionados, mais diretamente relacionados aos riscos ocupacionais, os hábitos de vida, como as atividades de lazer e as condições gerais de vida (níveis de escolaridade e renda), podem ter impactos sobre a saúde mental dos indivíduos (PATEL et al., 2003).

Considerando que, no Brasil, ainda é restrito o número de estudos epidemiológicos voltados para avaliação da saúde mental das populações trabalhadoras, de modo a estabelecer um quadro mais aproximado da situação de saúde mental nesses grupos e dos fatores associados às repercussões negativas sobre a saúde, este estudo, de caráter exploratório, objetivou estimar a ocorrência de transtornos mentais comuns (TMC) em trabalhadores, avaliando a ocorrência desses transtornos segundo diferentes aspectos da vida dos trabalhadores: características sociodemográficas, características do trabalho, atividades de lazer e aspectos psicossociais de trabalho. $\mathrm{O}$ estudo foi realizado com trabalhadores residentes na zona urbana da cidade de Feira de Santana, Bahia.

Espera-se que os resultados deste estudo possam subsidiar a reflexão sobre a situação de saúde mental dos trabalhadores e contribuir para a elaboração de projetos e políticas de saúde mental e para a gestão dos serviços de atenção à saúde e de segurança e ambiente das empresas, além de orientar ações sindicais em saúde voltadas a proporcionar melhores condições de trabalho e, consequentemente, de vida aos trabalhadores.

\section{Materiais e métodos}

Trata-se de um estudo epidemiológico de corte transversal com trabalhadores da zona urbana de Feira de Santana, Bahia, realizado a partir de um estudo de base populacional, desenvolvido pelo Núcleo de Epidemiologia da Universidade Estadual de Feira de Santana. Tem como finalidade investigar a situação de saúde mental da população urbana, com 15 anos ou mais de idade, de Feira de Santana, Bahia.

\section{População de estudo e critérios amostrais}

Para o estudo de base populacional, a amostra foi selecionada por procedimentos aleatórios. A seleção das áreas de estudo foi feita por amostragem estratificada por subdistritos, a partir de dados censitários do IBGE (INSTITUTO BRASILEIRO DE GEOGRAFIA E ESTATÍSTICA, 2000).

A amostragem foi feita por etapas múltiplas sucessivas, nas quais foram adotados os seguintes procedimentos: 1. determinação da representação percentual da população por subdistrito da zona urbana; 2. definição do percentual na amostra de cada subdistrito a partir dos dados de população residente em cada subdistrito; 3. listagem dos setores censitários em cada subdistrito; 4. seleção aleatória dos setores censitários a serem incluídos na amostra em cada subdistrito; 5. seleção aleatória das ruas a serem incluídas na amostra em cada setor censitário selecionado; 6. todos os domicílios nas ruas sorteadas foram incluídos na amostra; 7. todos os indivíduos com 15 anos ou mais de idade, residentes nos domicílios das ruas sorteadas, foram considerados indivíduos elegíveis para compor a amostra. Para reduzir o percentual de perdas, foi adotado o procedimento de realizar até três visitas por domicílio.

Para o cálculo do tamanho da amostra (projeto-mãe), assumiu-se estimativa de prevalência de TMC de 24\% (ORGANIZAÇÃO MUNDIAL DE SAÚDE, 2001), erro amostral de 3\%, com 95\% de confiança. Assumindo-se esses parâmetros, estabeleceu-se uma amostra de 800 indivíduos. Considerando-se o efeito do desenho do estudo (amostragem por conglomerado), dobrou-se o tamanho da amostra (1.600 pessoas). Admitindo-se recusas e perdas em torno de $20 \%$, definiu-se o tamanho da amostra em 1.920 indivíduos. Ao final do estudo, foram visitados 1.479 domicílios e entrevistadas 3.190 pessoas.

Para este estudo, foi feito um recorte do estudo populacional, analisando-se os dados somente da população trabalhadora. A definição de população trabalhadora foi feita a partir da resposta afirmativa à questão "você está trabalhando atualmente"? Portanto, considerou-se como trabalhador o indivíduo que referiu estar trabalhando no momento da coleta de dados, independentemente do tipo de vínculo de trabalho (formal ou informal), do setor de trabalho ou do valor da remuneração recebida.

Como o estudo não foi desenhado especificamente para estudar a população de trabalhadores de Feira de Santana, recalculou-se o $n$ amostral para verificar se este estudo teria poder suficiente para avaliar tal população. Para cálculo do tamanho da amostra dos trabalhadores, foram considerados: o número de trabalhadores de Feira de Santana estimado pelo Dieese (DEPARTAMENTO INTERSINDICAL DE ESTATÍSTICA E ESTUDO SOCIOECONÔMICOS, 2002) - 120.400 trabalhadores -, a prevalência de TMC de 24\% (ORGANIZAÇÃO MUNDIAL DE SAÚDE, 2001), erro amostral de 3\%, com 95\% de confiança. A partir desses parâmetros, chegou-se ao tamanho da amostra de 890 trabalhadores.

A amostra de trabalhadores investigada totalizou 1.311 indivíduos; alcançando, assim, um tamanho amostral superior ao estimado. 


\section{Instrumentos de coleta de dados}

Foram utilizados dois instrumentos estruturados para a coleta de dados: a ficha domiciliar, incluindo informações gerais do domicílio, e o questionário individual, contendo dados referentes a: características sociodemográficas (sexo, idade, escolaridade, entre outras), condições de vida (chefia da família, renda, tipo de moradia), hábitos de vida (prática de atividades físicas e de lazer), saúde reprodutiva, trabalho doméstico, atividades de lazer e atos de violência. A saúde mental foi avaliada pelo Self-Reporting Questionnaire (SRQ-20) e os aspectos psicossociais relacionados ao trabalho foram mensurados pelo Job Content Questionnaire (JCQ) (ARAÚJO; GRAÇA; ARAÚJO, 2003).

A entrevista, conduzida por pesquisadores treinados, foi realizada no domicílio do trabalhador.

\section{Variáveis de estudo}

Este estudo, de caráter exploratório, investigou diferentes aspectos da vida dos trabalhadores: características sociodemográficas (sexo, idade, escolaridade, situação conjugal, cor da pele autorreferida e renda média mensal), características do trabalho (tipo, setor e turno de trabalho, carga horária, número de dias de trabalho e ocupação), atividades de lazer (se realiza regularmente ou não tais atividades) e aspectos psicossociais de trabalho.

Para avaliação dos aspectos psicossociais do trabalho, foi utilizado o Job Content Questionnaire (JCQ), ou Questionário do Conteúdo do Trabalho (KARASEK, 1985; KARASEK; THEÖRELL, 1990). A versão recomendada do JCQ é composta por 49 questões incluindo mensuração de: controle sobre o próprio trabalho, demandas psicológicas, demandas físicas, suporte social no trabalho (da chefia e dos colegas) e insegurança no emprego. O JCQ é um questionário estruturado; as questões são avaliadas em escalas do tipo Likert variando de 1 (discordo fortemente) a 4 (concordo fortemente).

As dimensões de controle sobre o trabalho e de demandas psicológicas podem ser avaliadas simultaneamente em um modelo bidimensional: o modelo demanda-controle (KARASEK; THEÖRELL, 1990). Como já mencionado, neste modelo é proposta a análise do trabalho a partir da combinação de níveis de baixo e alto controle e de baixa e alta demanda psicológica. $\mathrm{O}$ trabalho em alta exigência é compreendido como a situação laboral de maior exposição ocupacional, configurando situações de risco para a saúde física e mental (KARASEK; THEÖRELL, 1990).

A versão em português do JCQ utilizada neste estudo foi a traduzida e validada por Araújo e Karasek (2008). O estudo de validação foi realizado em população de trabalhadores formais e informais e evidenciou bom desempenho global do JCQ de modo similar ao observado em outros estudos (KARASEK et al., 1998): a consistência interna, avaliada pelos coeficientes de Cronbah, foi satisfatória para as escalas avaliadas, e a análise fatorial mostrou uma estrutura consistente com os pressupostos do modelo teórico das escalas proposto.
Neste estudo, foram incluídas na análise as dimensões de controle e de demanda psicológica. Para o cálculo dessas escalas, foram seguidos os procedimentos do manual do JCQ (KARASEK, 1985). Em seguida, essas variáveis foram dicotomizadas, utilizando a média como ponto de corte. Os níveis de alto e baixo controle e de alta e baixa demanda foram combinados de modo a constituir os grupos do modelo demanda-controle: baixa exigência (alto controle e baixa demanda), trabalho passivo (baixo controle e baixa demanda), trabalho ativo (alto controle e alta demanda) e de alta exigência (baixo controle, alta demanda)

A variável resposta investigada foram os TMCs que são caracterizados, segundo Goldberg e Huxley (1992), por sinais e sintomas como: modificações do humor, irritabilidade, esquecimento, insônia, fadiga, agressividade, dificuldade de concentração e queixas psicossomáticas.

Os transtornos mentais comuns (TMC) foram mensurados pelo Self-Reporting Questionnaire (SRQ-20) - instrumento recomendado por ser autoaplicável, composto de 20 questões com respostas dicotômicas (sim/ não) para mensurar morbidade psíquica não psicótica (WHO, 1994). As questões do SRQ-20 são avaliadas considerando-se os últimos 30 dias. O SRQ é usado para detectar presença de sintomas em geral. Portanto, destina-se à caracterização de grau de suspeição de transtorno psíquico, não estabelecendo diagnóstico do tipo específico de distúrbio encontrado. Assim, é um instrumento que se destina a detecção de sintomas, sendo adequado para estudos de populações (MARI; WILLIAMS, 1986; SANTOS; ARAÚJO; FERNANDES, 2009).

No Brasil, o SRQ-20 foi traduzido e validado por Mari e Williams (1986), obtendo-se sensibilidade de $83 \%$, especificidade de $80 \%$ e taxa de classificação incorreta de $19 \%$.

O SRQ-20 tem sido um dos instrumentos mais utilizados nos estudos brasileiros para avaliação de saúde mental em populações trabalhadoras (LUDERMIR; MELO-FILHO, 2002; ARAÚJO et al., 2003; DELCOR et al., 2004; REIS et al., 2005; NASCIMENTO-SOBRINHO et al., 2006). No estudo realizado por Santos, Araújo e Oliveira (2009), observou-se desempenho satisfatório do SRQ-20 em investigações relacionadas à saúde mental de trabalhadores. Resultados dos estudos analisados por esses autores evidenciaram tratar-se de um instrumento consistente e com boa capacidade discriminatória: coeficiente de consistência interna geral e padronizado de 0,80 , estrutura fatorial compatível com uma variedade significativa de sintomatologia psíquica, permitindo identificar diferentes grupos de sintomas. Assim, o SRQ-20 apresenta desempenho aceitável para identificar transtornos mentais comuns, com boa capacidade para rastreamento de morbidade psíquica em população trabalhadora.

Neste estudo, para definição de transtornos mentais comuns, adotou-se, como ponto de corte, 7 (sete) ou mais respostas positivas, conforme utilizado em outros estudos (ARAÚJO et al., 2003; NASCIMENTO-SOBRINHO, 2006; PORTO et al., 2006). 


\section{Análise dos dados}

Procedeu-se inicialmente à caracterização da população segundo os aspectos de interesse: características sociodemográficas, do trabalho profissional, aspectos psicossociais do trabalho (modelo demanda-controle) e atividades de lazer.

Posteriormente, foi conduzida a análise univariada para examinar associações brutas das variáveis investigadas e a ocorrência de TMC. Foram calculadas as taxas de prevalência, razões de prevalência (RP) e seus respectivos intervalos de 95\% de confiança. Também foi utilizado o teste qui-quadrado para avaliação de associação entre as variáveis de interesse, adotando-se $\mathrm{p} \leq 0,05$ para associação estatisticamente significante. Em seguida, com o objetivo de avaliar o efeito simultâneo das variáveis estudadas, realizou-se análise de regressão logística múltipla (ARLM) de natureza exploratória.

O modelo completo, testado na ARLM, incluiu as variáveis: sexo, idade, cor da pele autorreferida (categorizada em raça negra, preta/parda, e não negra), situação conjugal, renda média mensal; características do trabalho: tipo de contrato de trabalho (formal e informal), carga horária diária de trabalho (até 8 horas/ mais de 8 horas), setor de trabalho (comércio, indústria, transporte, educação, serviços em geral e serviços domésticos), atividades de lazer (realiza/não realiza), ocupação e aspectos psicossociais do trabalho (demandas psicológicas e controle sobre o próprio trabalho).

A ARLM foi conduzida conforme os procedimentos recomendados por Hosmer e Lemeshow (2000) e incluiu as seguintes etapas: 1) Seleção das variáveis a partir dos objetivos do estudo e de revisão de literatura; 2) Verificação de pressupostos do modelo; 3) Pré-seleção das variáveis para inclusão na análise através do teste de razão de verossimilhança, adotando um valor de $\mathrm{p}$ $\leq 0,25$; 4) ARLM propriamente dita, utilizando procedimento backward. O critério de significância $\mathrm{p}<0,10$ foi adotado para obtenção do modelo final.

Considerando que a ARLM foi desenvolvida para análise de estudos de caso-controle, produzindo medidas de Odds ratio (OR) e não de Razão de Prevalência (RP), e que o efeito de interesse neste estudo (TMC) foi elevado, procedeu-se ao cálculo das estimativas das razões de prevalência (RP). Com base nos parâmetros do modelo final obtido, foram estimadas as RP e seus respectivos intervalos de confiança, utilizando o Método Delta, desenvolvido por Oliveira, Santana e Lopes (1997).

Os dados foram analisados utilizando os programas SPSS (Statistical Package for Social Sciences), versão 10.0, Epi Info versão 6.0 e o "R" The $R$ Foundation for Statistical Computing, versão 2.2.1.

A pesquisa foi encaminhada ao Comitê de Ética em Pesquisa da Universidade Estadual de Feira de Santana, seguindo os referenciais básicos da bioética e as especificações da Resolução 196/96 do Conselho Nacional de Saúde, tendo sido aprovada sob o protocolo 17/01.

\section{Resultados}

Na população estudada, evidenciou-se percentual similar de homens e mulheres, com ligeiro predomínio para o sexo masculino (50,2\%) (Tabela 1). A faixa etária predominante foi a de 20 a 39 anos (55,4\%), portanto, uma população relativamente jovem. A baixa escolaridade foi marcante: $43,5 \%$ dos trabalhadores relataram possuir o Ensino Fundamental e apenas 6,3\% o nível superior. Observou-se maior proporção de casados $(51,5 \%)$ e de trabalhadores que autorreferiram cor da pele parda/preta $(77,0 \%)$. Com relação ao rendimento mensal, verificou-se que $40,3 \%$ recebiam menos de um salário mínimo e 44,5\% ganhavam de um até três salários mínimos. Cerca de $1 / 3$ da população não participava de atividades regulares de lazer (Tabela 1).

Em relação às características do trabalho, o comércio foi o setor que mais empregou os trabalhadores estudados, 38,7\% do total, seguido pelo setor de serviços em geral (27,8\%) (Tabela 2). Como ocupação principal, predominaram os trabalhadores do comércio $(38,8 \%)$.

A maioria $(84,4 \%)$ referiu ter trabalho regular. O vínculo de trabalho mais expressivo foi o assalariado com e sem comissão $(35,1 \%)$, seguido do profissional autônomo (34,1\%). A maioria dos trabalhadores $(66,2 \%)$ trabalhava 6 (seis) ou mais dias na semana. Em relação ao turno de trabalho, $80,5 \%$ trabalhavam durante o dia e apenas $3,4 \%$ trabalhavam à noite. Identificou-se elevada jornada de trabalho: a carga horária semanal excedia às 8 horas diárias de trabalho para 38,7\%.

Com relação às características psicossociais do trabalho de acordo com o modelo demanda-controle, foi observado que as proporções mais elevadas de trabalhadores em situação de alta exigência foram observadas na construção civil $(34,8 \%)$, nos serviços domésticos $(30,6 \%)$ e no comércio $(27,1 \%)$ (Tabela 3). Em situação de baixa exigência, as proporções mais expressivas foram no setor de ensino (35,0\%), transporte $(20,2 \%)$ e serviços em geral $(29,1 \%)$. Os trabalhadores do ensino $(32,5 \%)$ e da indústria $(38,0 \%)$ também apresentaram proporções relevantes em trabalho ativo. Merece destaque o baixo percentual, em situação de trabalho ativo, dos trabalhadores de serviços domésticos (5,6\%). Para a situação de trabalho passivo, destacou-se a proporção do setor de serviços domésticos (40,3\%).

As maiores proporções de trabalho regular foram observadas em trabalho em baixa exigência e trabalho ativo; por outro lado, a maior proporção de atividade laboral temporária ou sazonal foi encontrada em alta exigência.

As proporções dos grupos de demanda-controle foram similares para trabalho diurno. Nos trabalhos noturno e de revezamento de turno, cabe destacar maiores proporções na situação de trabalho ativo (respectivamente $29,6 \%$ e $34,5 \%$ ).

As maiores proporções de carga horária de 8 horas diárias de trabalho foram encontradas no trabalho de baixa exigência $(28,8 \%)$ e mais de 8 horas diárias, no trabalho ativo $(30,0 \%)$ e no trabalho de alta exigência $(26,5 \%)$. 
As proporções entre o número de dias trabalhados (de 1 a 5 dias na semana ou 6 a 7 dias) foram similares entre os grupos estudados (Tabela 3).

As situações de trabalho entre os biscateiros e as empregadas domésticas foram similares: em ambas destacaram-se os percentuais em trabalho passivo (respectivamente $30,2 \%$ e $42,9 \%$ ) e em alta exigência
(28,1\% e $30,6 \%)$. O perfil também foi semelhante entre autônomos e profissionais liberais, para os quais foi mais frequente o trabalho em baixa exigência e o trabalho ativo.

Entre os assalariados e os funcionários públicos, destacou-se a situação de trabalho ativo $(27,4 \%$ e $36,1 \%$, respectivamente) (Tabela 3 ).

Tabela 1 Distribuição das características sociodemográficas dos trabalhadores estudados da zona urbana de Feira de Santana, Bahia, 2002

\begin{tabular}{|c|c|c|}
\hline Características sociodemográficas & $n$ & $\%$ \\
\hline \multicolumn{3}{|l|}{$\operatorname{Sexo}(N=1.311)$} \\
\hline Feminino & 653 & 49,8 \\
\hline Masculino & 658 & 50,2 \\
\hline \multicolumn{3}{|l|}{ Faixa etária $(\mathrm{N}=1.311)$} \\
\hline $15-19$ anos & 133 & 10,1 \\
\hline $20-29$ anos & 395 & 30,1 \\
\hline $30-39$ anos & 332 & 25,3 \\
\hline $40-49$ anos & 252 & 19,2 \\
\hline 50 anos ou mais & 199 & 15,2 \\
\hline \multicolumn{3}{|l|}{ Escolaridade $(\mathrm{N}=1.304)$} \\
\hline Analfabeto & 103 & 7,9 \\
\hline Ensino Fundamental & 567 & 43,5 \\
\hline Ensino Médio & 552 & 42,3 \\
\hline Superior & 82 & 6,3 \\
\hline \multicolumn{3}{|l|}{ Cor da pele $(\mathrm{N}=1.254)$} \\
\hline Branca & 276 & 22,0 \\
\hline Parda & 739 & 58,9 \\
\hline Preta & 227 & 18,1 \\
\hline Amarela/indígena & 12 & 1,0 \\
\hline \multicolumn{3}{|l|}{ Situação conjugal ( $\mathrm{N}=1.304)$} \\
\hline Casado/união livre & 672 & 51,5 \\
\hline Solteiro & 507 & 38,9 \\
\hline Viúvo & 36 & 2,8 \\
\hline Divorciado/desquitado/separado & 89 & 6,8 \\
\hline \multicolumn{3}{|l|}{ Renda $(\mathrm{N}=1.188)$} \\
\hline$<1$ salário & 479 & 40,3 \\
\hline 1 a 3 salários & 529 & 44,5 \\
\hline 3 a 5 salários & 108 & 9,1 \\
\hline$>5$ salários & 72 & 6,1 \\
\hline \multicolumn{3}{|l|}{ Atividades de lazer $(\mathrm{N}=1.310)$} \\
\hline Sim & 895 & 68,3 \\
\hline Não & 415 & 31,7 \\
\hline
\end{tabular}


Tabela 2 Características do trabalho profissional. Trabalhadores urbanos de Feira de Santana, Bahia, 2002

\begin{tabular}{|c|c|c|}
\hline Características do trabalho & $n$ & $\%$ \\
\hline \multicolumn{3}{|l|}{ Setor de trabalho $(\mathrm{N}=1.219)$} \\
\hline Serviços domésticos" & 137 & 1,2 \\
\hline Construção civil & 50 & 4,1 \\
\hline Indústria manufatureira & 80 & 6,6 \\
\hline Comércio & 473 & 38,8 \\
\hline Transporte & 56 & 4,6 \\
\hline Ensino & 84 & 6,9 \\
\hline Serviços em geral*** & 339 & 27,8 \\
\hline \multicolumn{3}{|l|}{ Tipo de trabalho $(\mathrm{N}=1.185)$} \\
\hline Regular & 1000 & 84,4 \\
\hline Temporário/sazonal & 185 & 15,7 \\
\hline \multicolumn{3}{|l|}{ Turno de trabalho $(\mathrm{N}=945)$} \\
\hline Diurno & 761 & 80,5 \\
\hline Noturno & 32 & 3,4 \\
\hline Revezamento & 152 & 16,1 \\
\hline \multicolumn{3}{|l|}{ Carga horária $(\mathrm{N}=1.311)$} \\
\hline Até 8 horas & 803 & 61,3 \\
\hline Mais de 8 horas & 508 & 38,7 \\
\hline \multicolumn{3}{|l|}{ Dias de trabalho $(\mathrm{N}=1.311)$} \\
\hline 1 a 5 dias & 443 & 33,8 \\
\hline 6 a 7 dias & 868 & 66,2 \\
\hline \multicolumn{3}{|l|}{ Vínculo de trabalho $(\mathrm{N}=1.127)$} \\
\hline Biscateiro & 110 & 9,8 \\
\hline Empregado doméstico & 110 & 9,8 \\
\hline Autônomo & 384 & 34,1 \\
\hline Profissional liberal & 23 & 2,0 \\
\hline Assalariado com e sem comissão & 397 & 35,1 \\
\hline Funcionário público & 90 & 8,0 \\
\hline Empregador/empresário & 13 & 1,2 \\
\hline
\end{tabular}

* Serviços domésticos incluiu todos os empregados que desenvolviam atividades laborais em domicílios, com ou sem vínculo formal de trabalho, tais como jardineiros, empregadas domésticas, babás, motoristas particulares, dentre outros.

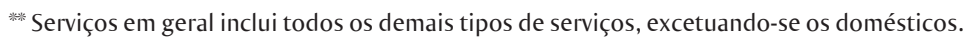

\section{Saúde mental dos trabalhadores}

A prevalência global de transtornos mentais comuns na população de trabalhadores foi de $25,2 \%$, sendo mais elevada entre as mulheres $(36,6 \%)$ do que entre os homens (13,8\%) (Tabela 4). A diferença observada foi estatisticamente significante.

Verificou-se que os trabalhadores mais velhos apresentaram maior prevalência de TMC $(32,2 \%)$, embora a diferença tenha sido estatisticamente significante apenas para o estrato de idade mais avan- çada (50 anos ou mais) quando comparado ao estrato mais jovem (15-19 anos). Com relação à escolaridade, observou-se que os trabalhadores com maior nível de escolaridade apresentaram menor prevalência de TMC (15,9\% entre aqueles de nível superior e 41,7\% entre os analfabetos).

Maior prevalência de TMC foi encontrada entre os trabalhadores de raça negra (preta $28,2 \%$ e parda $27,1 \%$ ). Os viúvos e divorciados/separados/desquitados apresentaram as prevalências mais elevadas de TMC $(36,1 \%)$. 
Tabela 3 Características do trabalho segundo os grupos do modelo Demanda-Controle entre trabalhadores urbanos de Feira de Santana, Bahia, 2002

\begin{tabular}{|c|c|c|c|c|c|c|c|c|}
\hline \multirow{3}{*}{ Características do trabalho } & \multicolumn{8}{|c|}{ Modelo demanda-controle } \\
\hline & \multicolumn{2}{|c|}{ Baixa exigência } & \multicolumn{2}{|c|}{ Trabalho ativo } & \multicolumn{2}{|c|}{ Trabalho passivo } & \multicolumn{2}{|c|}{ Alta exigência } \\
\hline & $n$ & $\%$ & $n$ & $\%$ & $n$ & $\%$ & $n$ & $\%$ \\
\hline \multicolumn{9}{|l|}{ Setor de trabalho } \\
\hline Serviços domésticos & 29 & 23,4 & 7 & 5,6 & 50 & 40,3 & 38 & 30,6 \\
\hline Construção civil & 12 & 26,1 & 13 & 28,3 & 5 & 10,9 & 16 & 34,8 \\
\hline Indústria manufatureira & 15 & 21,1 & 27 & 38,0 & 11 & 15,5 & 18 & 25,4 \\
\hline Comércio & 118 & 26,6 & 133 & 30,0 & 85 & 19,2 & 107 & 24,2 \\
\hline Transporte & 14 & 29,2 & 11 & 22,9 & 10 & 20,8 & 13 & 27,1 \\
\hline Ensino & 28 & 35,0 & 26 & 32,5 & 15 & 18,8 & 11 & 13,8 \\
\hline Serviços em geral & 91 & 29,1 & 91 & 29,1 & 76 & 24,3 & 55 & 17,6 \\
\hline \multicolumn{9}{|l|}{ Tipo de trabalho } \\
\hline Regular & 266 & 28,7 & 259 & 27,9 & 198 & 21,3 & 205 & 22,1 \\
\hline Temporário/sazonal & 39 & 22,8 & 42 & 24,6 & 44 & 25,7 & 46 & 26,9 \\
\hline \multicolumn{9}{|l|}{ Turno de trabalho } \\
\hline Diurno & 182 & 25,9 & 179 & 25,5 & 168 & 23,9 & 173 & 24,6 \\
\hline Noturno & 6 & 22,2 & 8 & 29,6 & 7 & 25,9 & 6 & 22,2 \\
\hline Revezamento & 27 & 18,6 & 50 & 34,5 & 36 & 24,8 & 32 & 22,1 \\
\hline \multicolumn{9}{|l|}{ Carga horária } \\
\hline Até 8 horas & 215 & 28,8 & 191 & 25,6 & 182 & 24,4 & 158 & 21,2 \\
\hline Mais de 8 horas & 105 & 23,0 & 137 & 30,0 & 94 & 20,6 & 121 & 26,5 \\
\hline \multicolumn{9}{|l|}{ Dias de trabalho } \\
\hline 1 a 5 dias & 100 & 24,6 & 121 & 29,8 & 96 & 23,6 & 89 & 21,9 \\
\hline 6 a 7 dias & 220 & 27,6 & 207 & 26,0 & 180 & 22,6 & 190 & 23,8 \\
\hline \multicolumn{9}{|l|}{ Vínculo de trabalho } \\
\hline Biscateiro & 22 & 22,9 & 18 & 18,8 & 29 & 30,2 & 27 & 28,1 \\
\hline Empregado doméstico & 22 & 22,4 & 4 & 4,1 & 42 & 42,9 & 30 & 30,6 \\
\hline Autônomo/profissional liberal & 126 & 33,9 & 133 & 35,7 & 37 & 9,9 & 76 & 20,4 \\
\hline Assalariado com e sem comissão & 88 & 23,4 & 103 & 27,4 & 100 & 26,7 & 84 & 22,4 \\
\hline Funcionário público & 21 & 25,3 & 30 & 36,1 & 18 & 21,7 & 14 & 16,9 \\
\hline Empregador/empresário & 3 & 23,1 & 2 & 15,4 & 6 & 46,2 & 2 & 15,4 \\
\hline
\end{tabular}

A baixa renda foi fator fortemente associado à ocorrência de TMC. A prevalência de TMC entre aqueles que tinham menos de um salário mínimo foi quatro vezes maior quando comparados com aqueles que recebiam mais de três salários mínimos.

A ausência de participação em atividades de lazer associou-se positivamente à prevalência de transtornos mentais comuns: trabalhadores que não mantinham regularmente atividades de lazer apresentaram prevalência de TMC duas vezes mais elevada do que aqueles que participavam regularmente dessas atividades.

Analisando a prevalência de TMC segundo características do trabalho, observaram-se diferenças de acordo com os setores de trabalho onde atuavam. No setor de serviços domésticos, a prevalência de TMC foi de $46,7 \%$ e, no setor de ensino, de $28,6 \%$, enquanto no setor de transporte foi de apenas $8,9 \%$. As diferenças observadas, tomando-se o setor de transporte como grupo de referência, foram estatisticamente significantes (Tabela 5).

Em relação ao vínculo de trabalho, os empregados domésticos $(50,9 \%)$ apresentaram a maior prevalência de TMC, enquanto entre os empregadores/empresários se registrou a menor $(15,4 \%)$.

Observou-se que trabalhadores submetidos à atividade temporária/sazonal apresentaram maior frequência de TMC $(61,4 \%)$ do que aqueles que tinham trabalho regular $(23,7 \%)$. Constatou-se também que aqueles que trabalhavam 6 a 7 dias por semana tinham menor prevalência de TMC $(24,1 \%)$ do que aqueles que trabalhavam um número menor de dias $(27,3 \%)$; mas a diferença observada não foi estatisticamente significante. Em relação à carga horária de trabalho, observou-se maior frequência de TMC $(29,8 \%)$ para aqueles que trabalhavam mais de 8 horas diárias; a diferença encontrada foi estatisticamente significante. 
Tabela 4 Prevalência (\%) de transtornos mentais comuns (TMC) segundo características sociodemográficas entre trabalhadores da zona urbana de Feira de Santana-BA, 2002

\begin{tabular}{|c|c|c|c|c|}
\hline Características & $P(\%)$ & $R P$ & IC95\% & Valorde $p$ \\
\hline TMC & 25,2 & - & - & - \\
\hline \multicolumn{5}{|l|}{ Sexo } \\
\hline Feminino & 36,6 & 2,65 & $(2,13-3,28)$ & $<0,001$ \\
\hline Masculino & 13,8 & $*$ & $*$ & $*$ \\
\hline \multicolumn{5}{|l|}{ Faixa etária } \\
\hline $15-19$ anos & 20,3 & $*$ & $*$ & $*$ \\
\hline $20-29$ anos & 22,5 & 1,11 & $(0,76-1,63)$ & 0,597 \\
\hline $30-39$ anos & 27,4 & 1,35 & $(0,92-1,97)$ & 0,111 \\
\hline $40-49$ anos & 23,4 & 1,15 & $(0,77-1,73)$ & 0,486 \\
\hline 50 anos ou mais & 32,2 & 1,58 & $(1,07-2,35)$ & 0,017 \\
\hline \multicolumn{5}{|l|}{ Escolaridade } \\
\hline Analfabeto & 41,7 & 2,63 & $(1,52-4,56)$ & $<0,001$ \\
\hline Ensino fundamental & 31,0 & 1,96 & $(1,17-3,27)$ & 0,004 \\
\hline Ensino médio & 17,2 & 1,09 & $(0,64-0,85)$ & 0,760 \\
\hline Superior & 15,9 & * & $*$ & $*$ \\
\hline \multicolumn{5}{|l|}{ Cor da pele } \\
\hline Branca & 18,1 & * & $*$ & * \\
\hline Parda & 27,1 & 1,49 & $(1,13-1,97)$ & $<0,003$ \\
\hline Preta & 28,2 & 1,56 & $(1,12-2,16)$ & $<0,007$ \\
\hline Amarela/indígena & 25,0 & 1,38 & $(0,50-3,79)$ & 0,824 \\
\hline \multicolumn{5}{|l|}{ Situação conjugal } \\
\hline Solteiro & 22,9 & $*$ & $*$ & $*$ \\
\hline Casado/união livre & 25,0 & 1,34 & $(1,08-1,65)$ & $<0,006$ \\
\hline Viúvo & 36,1 & 1,93 & $(1,21-3,07)$ & 0,010 \\
\hline Divorciado/desquitado & 36,0 & 1,92 & $(1,39-2,65)$ & $<0,001$ \\
\hline \multicolumn{5}{|l|}{ Renda } \\
\hline$<1$ salário & 38,8 & 3,88 & $(2,47-6,10)$ & $<0,001$ \\
\hline 1 a 3 salários & 17,2 & 1,72 & $(1,07-2,77)$ & 0,020 \\
\hline >3 salários & 10,0 & $*$ & * & $*$ \\
\hline \multicolumn{5}{|l|}{ Atividades de lazer } \\
\hline Sim & 18,9 & * & $*$ & $*$ \\
\hline Não & 38,8 & 2,05 & $(1,71-2,46)$ & $<0,001$ \\
\hline
\end{tabular}

TMC = Transtorno mentais comuns; $\mathrm{P}=$ prevalência; $\mathrm{RP}=$ razão de prevalência; IC95\% = intervalo de confiança de $95 \%$.

* = Grupo de referência.

A diferença observada para as prevalências de transtornos mentais comuns segundo níveis de demanda psicológica também foi estatisticamente significante: os trabalhadores com alta demanda psicológica apresentaram prevalência $23 \%$ maior do que aqueles em baixa demanda (Tabela 5). A prevalência de TMC entre os trabalhadores que referiram baixo controle sobre o próprio trabalho também foi maior do que entre aqueles com alto controle: prevalência 1,3 vez maior no primeiro grupo comparado ao segundo, a níveis estatisticamente significantes.

De acordo com os aspectos psicossociais, identificou-se maior prevalência de TMC no trabalho de alta exigência $(33,0 \%)$ quando comparado ao trabalho de baixa exigência. Essa diferença foi estatisticamente significante.
Na análise de regressão logística, permaneceram no modelo final as seguintes variáveis: escolaridade, renda, sexo, demandas do trabalho e atividades regulares de lazer. Assim, a exposição ao trabalho de alta demanda psicológica, com baixa renda $(\leq 3$ salários mínimos), baixo nível de escolaridade (sem instrução e Ensino Fundamental), do sexo feminino e que não praticavam atividades regulares de lazer foram fatores associados à ocorrência de transtornos mentais comuns. A força de associação entre TMC e as variáveis retidas no modelo final variou de 1,35 para a variável ausência de atividade de lazer (IC 95\%: $1,10-1,66$ ) a 3,04 vezes para o sexo feminino (IC $95 \%$ 2,34-3,95) (Tabela 6). 
Tabela 5 Prevalência (\%) de TMC segundo características do trabalho. Trabalhadores da zona urbana de Feira de Santana-BA, 2002

\begin{tabular}{|c|c|c|c|c|}
\hline Característica do trabalho & $P(\%)$ & $R P$ & IC 95\% & Valor de $p$ \\
\hline \multicolumn{5}{|l|}{ Setor de trabalho } \\
\hline Serviços domésticos & 46,7 & 5,23 & $(2,22-12,31)$ & $<0,001$ \\
\hline Construção civil & 26,0 & 2,91 & $(1,12-7,47)$ & 0,019 \\
\hline Indústria manufatureira & 25,0 & 2,0 & $(1,12-7,02)$ & 0,017 \\
\hline Comércio & 25,4 & 2,84 & $(1,21-6,65)$ & 0,006 \\
\hline Outros serviços & 18,3 & 2,05 & $(0,86-4,87)$ & 0,083 \\
\hline Ensino & 28,6 & 3,20 & $(1,30-7,89)$ & 0,004 \\
\hline Transporte & 8,9 & $*$ & $*$ & $*$ \\
\hline \multicolumn{5}{|l|}{ Tipo de trabalho } \\
\hline Regular & 23,7 & $*$ & $*$ & $*$ \\
\hline Temporário/sazonal & 61,4 & 1,28 & $(1,00-1,63)$ & 0,05 \\
\hline \multicolumn{5}{|l|}{ Vínculo de trabalho } \\
\hline Biscateiro & 26,4 & 1,71 & $(0,46-6,37)$ & 0,38 \\
\hline Autônomo & 27,3 & 1,78 & $(0,49-6,43)$ & 0,34 \\
\hline Profissional liberal & 8,7 & 0,57 & $(0,09-3,55)$ & 0,54 \\
\hline Assalariado com e sem comissão & 18,4 & 1,20 & $(0,33-4,35)$ & 0,78 \\
\hline Empregado doméstico & 50,9 & 3,31 & $(0,91-2,00)$ & 0,01 \\
\hline Funcionário público & 25,6 & 1,66 & $(0,44-6,24)$ & 0,42 \\
\hline Empregador/empresário & 15,4 & * & * & $*$ \\
\hline \multicolumn{5}{|l|}{ Dias trabalhados por semana } \\
\hline 1 a 5 dias & 27,3 & $*$ & $*$ & $*$ \\
\hline $6-7$ dias & 24,1 & 0,88 & $(0,73-1,07)$ & 0,20 \\
\hline \multicolumn{5}{|l|}{ Carga horária } \\
\hline Até 8 horas & 22,4 & $*$ & $*$ & * \\
\hline Mais de 8 horas & 29,8 & 1,33 & $(1,09-1,61)$ & 0,004 \\
\hline \multicolumn{5}{|l|}{ Turno de trabalho } \\
\hline Diurno/revezamento & 23,0 & $*$ & * & * \\
\hline Noturno & 18,5 & 0,80 & $(0,36-1,79)$ & 0,58 \\
\hline \multicolumn{5}{|l|}{ Demanda psicológica } \\
\hline Baixa & 22,6 & $*$ & $*$ & $*$ \\
\hline Alta & 27,7 & 1,23 & $(1,01-1,49)$ & 0,03 \\
\hline \multicolumn{5}{|l|}{ Controle } \\
\hline Baixo & 28,6 & 1,3 & $(1,07-1,58)$ & $<0,01$ \\
\hline Alto & 22,0 & $*$ & * & $*$ \\
\hline \multicolumn{5}{|l|}{ Grupos de demanda-controle } \\
\hline Baixa exigência & 20,3 & * & * & $*$ \\
\hline Trabalho ativo & 24,1 & 1,19 & $(0,88-1,58)$ & 0,248 \\
\hline Trabalho passivo & 24,3 & 1,20 & $(0,88-1,61)$ & 0,245 \\
\hline Alta exigência & 33,0 & 1,62 & $(1,23-2,14)$ & $<0,001$ \\
\hline
\end{tabular}

$\mathrm{P}=$ prevalência; $\mathrm{RP}=$ razão de prevalência; IC95\% = intervalo de confiança de 95\%.

* = Grupo de referência. 
Tabela 6 Razões de prevalências ajustadas, com seus respectivos intervalos de confiança (95\%), entre características estudadas e transtornos mentais comuns, obtidas na análise de regressão logística múltipla

\begin{tabular}{lcccc}
\hline \multicolumn{1}{c}{ Variáveis (exposto) } & RP bruta & IC95\% & RPajustada* $^{*}$ & IC95\% \\
\hline Sexo (feminino) & 2,65 & $(2,13-3,28)$ & 3,04 & $(2,34-3,95)$ \\
Escolaridade (baixa) ${ }^{* * *}$ & 1,89 & $(1,53-2,34)$ & 2,02 & $(1,57-2,60)$ \\
Renda (baixa) & 2,54 & $(1,62-3,99)$ & 1,78 & $(1,12-2,84)$ \\
Demanda psicológica (alta) & 1,23 & $(1,01-1,49)$ & 1,45 & $(1,14-1,84)$ \\
Lazer (ausência) & 2,05 & $(1,71-2,46)$ & 1,35 & $(1,10-1,66)$ \\
\hline
\end{tabular}

* Ajuste simultâneo das variáveis que permaneceram no modelo final de análise.

*** Baixa escolaridade: analfabeto e Ensino Fundamental; alta: Ensinos Médio e Superior.

****** Baixa renda: $\leq 3$ salários mínimos; Renda alta: > 3 salários mínimos.

\section{Discussão}

Este estudo descreveu o perfil de trabalhadores da zona urbana de Feira de Santana segundo características sociodemográficas, do trabalho, aspectos psicossociais do trabalho e atividades de lazer e estimou a prevalência de TMC segundo essas características.

Os trabalhadores estudados, em sua maioria, constituíam uma população relativamente jovem, com idade entre 20 e 39 anos, com baixa escolaridade, com predominância de níveis baixos de renda e composta por assalariados com e sem comissão $(35,1 \%)$ e por trabalhadores autônomos (34,1\%). O setor de comércio foi responsável pelo emprego de quase $40 \%$ da população trabalhadora amostrada, evidenciando que este setor, ao lado do setor de serviços em geral, é um dos mais importantes da economia do local estudado. Dados do IBGE (INSTITUTO BRASILEIRO DE GEOGRAFIA E ESTATÍSTICA, 2001) sobre a economia de Feira de Santana corroboram o perfil de emprego observado.

O perfil de baixa escolaridade observado entre os trabalhadores de Feira de Santana é similar ao perfil encontrado na Bahia. Estudo realizado por Souza (2010), utilizando os dados da Pesquisa Nacional por Amostras de Domicílios (PNAD), realizada pelo IBGE em 2001, apresenta informações sobre os níveis de escolaridade da Bahia e do Brasil. Na Bahia, 22\% dos trabalhadores não tinham nenhuma instrução ou tinham menos de um ano de estudo, o dobro do percentual verificado no país nesse nível de escolaridade. Além disso, na Bahia, 31,3\% dos trabalhadores apresentaram oito ou mais anos de estudo, enquanto no Brasil esse percentual representava $45,5 \%$ do total. Os dados evidenciaram também que na Bahia apenas 2,8\% tinham quinze anos ou mais de estudo; no Brasil, o percentual foi de 6,7\%. Os resultados desse estudo demonstraram que, em comparação com o Brasil, a população de trabalhadores do estado da Bahia ainda apresenta percentuais elevados nos níveis baixos de escolaridade (sem instrução ou de nível fundamental). Observa-se, portanto, que os dados obtidos neste estudo assemelham-se ao perfil do Estado como um todo.
O perfil de renda média mensal observada em Feira de Santana, com concentração nos estratos de baixa renda, é similar ao padrão do Estado. Os dados da PNAD, de 2001 (SOUZA, 2010), apontaram que no estrato de renda até um salário encontravam-se 38,3\% da população economicamente ativa da Bahia, enquanto no Brasil esse percentual era bem menor: $21,8 \%$. Por outro lado, nos estratos de renda acima de cinco salários mínimos estavam apenas 4,8\% dos trabalhadores na Bahia, enquanto no Brasil alcançava 13,0\% (percentual 2,7 vezes maior). Portanto, de modo similar ao observado com relação à escolaridade, observam-se claras desvantagens e atrasos dos trabalhadores da Bahia e de Feira de Santana com relação aos indicadores do país.

A prevalência global de TMC foi relevante: atingiu mais de um quarto da população estudada. Outros estudos, cujos desfechos foram os transtornos mentais comuns em trabalhadores, mensurados pelo SRQ-20, também evidenciaram que os problemas relativos à saúde mental são significativos em algumas populações de trabalhadores. No estudo entre motoristas e cobradores de ônibus urbanos da cidade de São Paulo, realizado por Souza e Silva (1998), observou-se prevalência de 20,3\%. Araújo et al. (2003) identificaram prevalência de distúrbios psíquicos menores de 33,3\% entre trabalhadoras de enfermagem num hospital público de Salvador-BA. Delcor et al. (2004) encontraram, entre professores da rede particular de ensino de Vitória da Conquista, Bahia, prevalência de distúrbios psíquicos menores de 45,2\%, enquanto Nascimento-Sobrinho et al. (2006) encontraram prevalência de 26,0\% entre médicos de Salvador.

Dentre as variáveis que foram analisadas, o sexo, a escolaridade, a renda, o trabalho em alta demanda psicológica e a ausência de atividades regulares de lazer estavam associadas à prevalência de transtornos mentais comuns. Esses são fatores que merecem atenção especial e demandam reflexão sobre as medidas a serem tomadas como alternativas possíveis para intervir na situação observada de modo a reduzir seu potencial nocivo à saúde. 
Neste estudo, a variável sexo esteve fortemente associada aos TMCs. As mulheres apresentaram prevalência 3 vezes maior de TMC do que os homens. Resultados semelhantes foram encontrados nos estudos de Reis et al. (2004), ao investigarem fatores associados aos TMCs, e de Brant (2004), que constatou associação entre gênero e sofrimento (as mulheres apresentaram prevalência 6 vezes maior de sofrimento psíquico do que os homens).

Estudos que avaliaram associação entre características do trabalho e saúde em mulheres destacam que as características dos ambientes e dos processos de trabalho são definidas pelas relações de gênero, configurando diferentes experiências de trabalho para os homens e para as mulheres (HALL, 1992); os estudos apontam expressivas desvantagens para as mulheres (ARAÚJO et al., 2006). Essas desvantagens associam-se aos impactos negativos sobre a saúde mental (GRIFFIN et al., 2002), especialmente se considerarmos que as mulheres trabalhadoras no Brasil permanecem como as principais responsáveis pelo trabalho doméstico e cuidado da família, elevando a carga total de trabalho para as mulheres que, simultaneamente, desempenham os papéis de mãe, mulher, esposa e trabalhadora (ARAÚJO et al., 2003).

Observou-se associação entre nível de escolaridade e TMC. Há evidências na literatura de que o analfabetismo e o baixo nível de escolaridade são fatores que favorecem transtornos mentais (PATEL; KLEINMAN, 2003). Ludermir e Melo-Filho (2002) também constataram elevadas prevalências de TMC nos grupos de nível fundamental e analfabeto. Maragno et al. (2006), num estudo de prevalência de TMC e variáveis sociodemográficas, constataram uma tendência linear de aumento de TMC em função do menor grau de escolaridade.

Diversos autores (LUDERMIR; MELO-FILHO, 2002; KLEINMAN; PATEL, 2003;) destacam que o nível de escolaridade proporciona aos indivíduos melhores condições de vida, tanto nos aspectos socioeconômicos, como na inserção na estrutura ocupacional. Além disso, influencia também nos aspectos relacionados à vida pessoal, como motivações, realização de sonhos, autoestima, melhores perspectivas futuras e, fundamentalmente, a busca de novos conhecimentos. De acordo com Ludermir e Melo-Filho (2002, p. 8):

a educação tem um efeito direto na saúde psicológica, pois aumenta a possibilidade de escolhas na vida e influencia aspirações, autoestima e aquisição de novos conhecimentos que podem motivar atitudes e comportamentos mais saudáveis.

A renda esteve associada aos TMCs. Lopes et al. (2003) encontraram associação entre a presença de TMC e renda. As dificuldades financeiras podem levar ao estresse e à insegurança. O baixo nível de escolaridade pode ser fator determinante para que os trabalhadores com menor oportunidade aceitem os baixos salários ou estejam inseridos no mercado informal de trabalho (PATEL; KLEINMAN, 2003).
O município de Feira de Santana, pela sua localização geográfica, como entroncamento rodoviário, favorece o grande fluxo migratório de indivíduos de regiões mais pobres para o município. Segundo Araújo (2001), esta situação geográfica facilita a migração crescente de pessoas que, na sua maioria, são desprovidas de renda e instrução. Estas evidências são confirmadas por Queiroz et al. (2004), ao observarem que a migração é um fator que tem corroborado para a degradação ambiental e o aumento do contingente populacional de baixa renda no município estudado.

Além das variáveis sociodemográficas que mostraram associação com TMC, atividade de lazer também foi um fator associado à ocorrência dos transtornos mentais investigados.

As atividades de lazer são importantes fatores de distensionamento e contribuem para a saúde física, mental e social das pessoas. Os indivíduos que participam de atividades de lazer coletivamente estabelecem relações afetivas entre si e com o mundo, possibilitando melhores condições de saúde, socialização e interesse pela vida, conforme destacam Almeida et al. (2005). Os achados de estudo realizados por Pondé e Caroso (2003) evidenciaram que o lazer, como atividade sociocultural, tem importante papel na saúde mental das pessoas, constituindo-se como fator de proteção da saúde mental.

A ausência de tempo livre, em função da carga de trabalho e da dupla jornada ocupacional, e falta de condição financeira são aspectos que comprometem o acesso ao lazer. A elevada carga de trabalho semanal a que estão submetidos os trabalhadores que, para sobreviverem, são obrigados a exercer várias atividades, como é frequentemente observado entre vigilantes, professores e profissionais de saúde (médicos, enfermeiros, dentistas, psicólogos), acaba por consumir o tempo que poderia ser dedicado a lazer, repouso e descanso (PONDÉ; CAROSO, 2003). Estudo realizado por Araújo, Graça e Araújo (2003) com o objetivo de descrever as prevalências de distúrbios psíquicos menores em professores universitários e dentistas constatou que cerca de $61 \%$ dos professores dedicavam 35 horas semanais às atividades profissionais e que os cirurgiões-dentistas (50\%) tinham carga horária semanal de trabalho variando de 36 a 44 horas. O duplo emprego foi referido por $47 \%$ dos professores, enquanto $42,3 \%$ dos dentistas relataram possuir dois ou mais empregos. A manutenção de múltiplos empregos é também uma característica frequente entre professores (DELCOR et al., 2004), trabalhadoras de enfermagem (ARAÚJO et al., 2003) e médicos (NASCIMENTO-SOBRINHO et al., 2006).

Estudo realizado por Almeida et al. (2005) sobre atividades de lazer entre idosos em Feira de Santana identificou que a cidade é desprovida de espaços de lazer para a população, em especial para os idosos. Constatou que os equipamentos de lazer são poucos e os existentes estão localizados no centro da cidade e se destinam às práticas físico-esportivas; portanto, não é oferecida à periferia da cidade qualquer possibilidade cultural ou 
de lazer. Desse modo, as opções encontradas para aqueles que podem consumir limita-se a frequentar bares e restaurantes. A inexistência ou as dificuldades de acesso aos equipamentos coletivos (praças, parques, ginásio poliesportivos, por exemplo) são fatores que impossibilitam à população, sobretudo a periférica, de participar de atividades de lazer. Políticas públicas voltadas para o aumento desses equipamentos devem ser pensadas como estratégias para o incremento da inserção em atividades de lazer, o que pode contribuir para elevação da qualidade de vida das populações.

Altos níveis de demandas psicológicas elevaram a prevalência de transtornos mentais comuns. Esse resultado é coerente com os achados de outros estudos que constataram associação entre alta demanda psicológica do trabalho e adoecimento psíquico, incluindo estudos longitudinais (STANSFELD et al., 1999). Reis et al. (2005) constataram elevada prevalência de distúrbios psíquicos menores em professores da rede municipal de Vitória da Conquista/ BA: professores expostos ao trabalho de alta demanda psicológica apresentaram prevalência 39\% maior do que trabalhadores em situação de baixa demanda. No estudo de Araújo, Graça e Araújo (2003), a prevalência de distúrbios psíquicos menores entre professores universitários e entre cirurgiões-dentistas foi mais elevada na situação de trabalho de alta demanda. Nascimento-Sobrinho et al. (2006), em estudo realizado com médicos em Salvador, também constataram maior prevalência para os médicos que referiram alta demanda.

Como se pode observar, a literatura registra resultados consistentes de associação entre elevada demanda psicológica e efeitos negativos sobre a saúde mental. A demanda psicológica tem um papel relevante na produção de sofrimento psíquico. Alguns estudos apontam que elevadas demandas psicológicas podem, inclusive, reduzir os efeitos benéficos de outros fatores, como, por exemplo, do alto controle sobre o próprio trabalho (ARAÚJO et al., 2003). Ou seja, quando as demandas são excessivas, o controle pode não promover os efeitos positivos esperados, porque o repertório e as possibilidades de responder adequadamente às demandas ambientais tornam-se insuficientes em função do nível de demanda encontrado: nessas situa- ções, o trabalhador não é mais capaz de responder como desejaria, o que gera uma situação de inadequação e intenso sofrimento. Esse sentimento pode ser a conexão entre essa exposição no ambiente de trabalho e a ocorrência de transtornos mentais. O ritmo, o volume e o tempo para se realizar o trabalho (indicadores que entram na avaliação da demanda psicológica aqui analisada) regem o modo operatório, o modo como se realiza o trabalho. Esses aspectos podem estruturar exposições significativas à saúde (SCHNALL; DOBSON; ROSSKAM, 2009).

Os resultados aqui obtidos devem ser avaliados com cautela em função de possíveis limitações do estudo. Dentre essas limitações, cabe considerar aquelas relativas ao tipo de desenho do estudo utilizado: corte transversal. Pode ter havido viés de seleção, pois se estudou apenas quem estava trabalhando no momento da pesquisa. Portanto, quem adoeceu e perdeu o emprego não entrou no estudo (viés de sobrevivência). Também não se pode descartar a hipótese de causa reversa, ou seja, não é possível identificar se os TMCs influenciaram os fatores associados ou vice-versa. O caráter transversal do estudo impossibilita análise de antecedência temporal.

Apesar dessas limitações, o estudo avaliou um contingente expressivo de trabalhadores, incluindo múltiplas categorias profissionais, vários setores da economia, com vínculo de trabalho formal e informal; ou seja, avaliou-se um conjunto bastante diversificado de trabalhadores. Além disso, os procedimentos de amostragem foram aleatórios e os instrumentos de pesquisa utilizados são largamente usados em estudos ocupacionais, revelando bom desempenho desses instrumentos em medir o que se propõe a medir.

Espera-se que os achados encontrados neste estudo contribuam para o melhor dimensionamento das questões relativas à saúde mental dos trabalhadores e para fomentar a discussão sobre os fatores associados ao adoecimento mental identificado. Assim, esta investigação pretendeu contribuir para um novo olhar sobre a saúde mental e o trabalho para que se possa, a partir daí, incentivar a proposição de medidas e ações que promovam a saúde física e mental dos trabalhadores.

\section{Contribuições de autoria}

Farias, M. D.: Realizou o delineamento e o planejamento do manuscrito, a análise dos dados e participou da elaboração, revisão e versão final do manuscrito; Araújo, T. M. de: Coordenou o projeto de pesquisa, participou da concepção do estudo, da coleta de dados, da análise dos dados, da elaboração, revisão e versão final do manuscrito.

\section{Referências}

ALMEIDA, M. M. G. et al. Atividades de lazer entre idosos. Revista Baiana de Saúde Pública, Salvador, v. 29, n. 2, p. 339-352, jul./dez. 2005.

ARAÚJO, E. M. Configuração do espaço urbano de Feira de Santana-BA. Revista de Saúde Coletiva da UEFS, Feira de Santana, v. 1, n. 1, p. 9-16, 2001.
ARAÚJO, T. M. et al. Aspectos psicossociais do trabalho e distúrbios psíquicos entre trabalhadoras de enfermagem. Revista de Saúde Pública, São Paulo, v. 37, n. 4, p. 424-433, 2003.

ARAÚJO, T. M.; GRAÇA, C. C.; ARAÚJO, E. M. Estresse ocupacional e saúde: contribuições do modelo 
demanda-controle. Ciência \& Saúde Coletiva, Rio de Janeiro, v. 8, n. 4, p. 991-1003, 2003.

ARAÚJO, T. M. et al. Diferenciais de gênero no trabalho docente e repercussões sobre a saúde. Ciência \& Saúde Coletiva, Rio de Janeiro, v. 11, n. 4, p. 11171129, 2006.

ARAÚJO, T. M.; KARASEK, R. Validity and reliability of the job content questionnaire in formal and informal jobs in Brazil. Scandinavian Journal of Work Environment \& Health, Helsinki, p. 52-59, 2008. Suplemento 6.

BRANT, L. C.; DIAS, E. C. Trabalho e sofrimento em gestores de uma empresa pública em reestruturação. Cadernos de Saúde Pública, Rio de Janeiro, v. 20, n. 4, p. 942-949, jul./ago. 2004.

CHECKOWAY, H.; PEARCE, N.; KRIEBEL, D. Research methods in occupational epidemiology. 2th ed. New York: Oxford University Press, 2004.

DEJOURS, C. A loucura do trabalho. 3. ed. São Paulo: Cortez, 1992.

DELCOR, N. S. et al. Condições de trabalho e saúde dos professores da rede particular de ensino de Vitória da Conquista, Bahia, Brasil. Cadernos de Saúde Pública, Rio de Janeiro, v. 20, n. 1, p. 187-196, jan./fev. 2004.

DEPARTAMENTO INTERSINDICAL DE ESTATÍSTICA E ESTUDO SOCIOECONÔMICOS. PED. Região metropolitana de Salvador. Disponível em: <http:// www.dieese.org.br/ped/ssa/pedssa/2002.pdf > . Acesso em: 22 ago. 2006.

FRANÇA, A. C. L.; RODRIGUES A. L. Stress e trabalho: uma abordagem psicossomática. 3. ed. São Paulo: Atlas, 2002.

GIATTI, L.; BARRETO, S. M. Situação do indivíduo no mercado de trabalho e iniqüidade em saúde no Brasil. Revista de Saúde Pública, São Paulo, v. 40, n. 1, p. 99106, 2006.

GRIFFIN, J. M. et al. The importance of low control at work and home on depression and anxiety: do these effects vary by gender and social class? Social Science $\&$ Medicine, v. 54, n. 5, p. 783-798, 2002.

GOLDENBERG, D.; HUXLEY. P. Common mental disorders: a bio-social model. London: Tavistock, 1992.

HALL, E. Double exposure: the combined impact of the home and work environments of psychosomatic strain in Swedish women and men. International Journal of Health Services, v. 22, n. 2, p. 239-260, 1992.

HOSMER, D. W.; LEMESHOW, S. Applied logistic regression. 2th. ed. New York: John Wiley \& Sons, 2000. \&

\section{INSTITUTO BRASILEIRO DE GEOGRAFIA E}

ESTATÍSTICA. Censo demográfico 2000: agregado por setores censitários dos resultados do universo. 2. ed. Disponível em: <ftp://ftp.ibge.gov.br/Censos/Censo_
Demografico_2000/Dados_do_Universo/Agregados por_Setores_Censitarios>. Acesso em: 15 maio 2005.

Pesquisa nacional por amostras de domicílios $\overline{P N A D,}$ 2001. Disponível em: < http://www.ibge.gov.br> Acesso em: 29 set. 2010.

KARASEK, R. A. Job demand, job decision latitude, and mental strain: implications for job redesign. Administrative Science Quarterly, v. 24, n. 2, p. 285308, 1979.

Job content questionnaire and user's guide. Massachusetts: University of Massachusetts, 1985. KARASEK, R. A.; THEÖRELL, T. Healthy work-stress, productivity, and the reconstruction of working life. New York: Basic Books, 1990.

KARASEK, R. A. et al. The Job Content Questionnaire (JCQ): an instrument for internationally comparative assessment of psychosocial job characteristics. Journal of Occupational Health Psychology, v. 3, n. 4, p. 322-355, 1998.

LAURELL, A. C; NORIEGA, M. Processo de produção e saúde: trabalho e desgaste operário. São Paulo: Hucitec, 1989.

LOPES, C. S.; FAERSTEIN, E.; CHOR, D. Eventos de vida produtores de estresse e transtornos mentais comuns: resultados do Estudo Pró-Saúde. Cadernos de Saúde Pública, Rio de Janeiro, v. 19, n. 6, p. 7131720, nov. 2003.

LUDERMIR, A. B.; MELO FILHO, D. A. Condições de vida e estrutura ocupacional associadas a transtornos mentais comuns. Revista de Saúde Pública, São Paulo, v. 36, n. 2, p. 213-221, jan. 2002.

MARAGNO, L. et al. Prevalência de transtornos mentais comuns em populações atendidas pelo programa Saúde da Família (QUALIS) no município de São Paulo, Brasil. Cadernos de Saúde Pública, Rio de Janeiro, v. 22, n. 8, p. 1639-1648, ago. 2006.

MARI, J. J.; WILLIAMS, P. A validity study of a psychiatric screening questionnaire (SRQ-20) in primary care in the city of Sao Paulo. British Journal of Psychiatry, Londres, v. 148, n. 1. p. 23-26, 1986.

NASCIMENTO-SOBRINHO, C. L. et al. Condições de trabalho e saúde mental dos médicos de Salvador, Bahia, Brasil. Cadernos de Saúde Pública, Rio de Janeiro, v. 22, n. 2, p. 131-140, jan. 2006.

OLIVEIRA, N. F.; SANTANA V. S.; LOPES, A. A. Razões de proporções e uso do método Delta para intervalos de confiança em regressão logística. Revista de Saúde Pública, v. 31, n. 1, p. 90-99, fev. 1997.

ORGANIZAÇÃO MUNDIAL DE SAÚDE. Relatório mundial da saúde. Saúde mental: nova concepção, nova esperança. Lisboa: Direção Geral da Saúde, 2001. Disponível em: <http: //www.opas.org.br/ promocao/uploadArqsmental.pdf $>$. Acesso em: 26 fev. 2007. 
PATEL, V.; KLEINMAN, A. Poverty and common mental disorders in developing countries. Bulletin of the World Health Organization, v. 81, n. 8, p. 606-615, 2003.

PONDÉ, M. P.; CAROSO, C. Lazer como fator de proteção da saúde mental. Revista de Ciências Médicas, v. 12, n. 2, p. 163-172, abr./jun. 2003.

PORTO, L. A. et al. Associação entre distúrbios psíquicos e aspectos psicossociais do trabalho de professores. Revista de Saúde Pública, São Paulo, v. 40, n. 5, p. 818-826, 2006.

POSSAS, C. Epidemiologia e SociedadeHeterogeneidade estrutural e saúde no Brasil. São Paulo: Hucitec, 1989.

QUEIROZ, C. M. B. et al. Qualidade de vida e políticas públicas no município de Feira de Santana. Ciência \& Saúde Coletiva, Rio de Janeiro, v. 9, n. 2, p. 411-421, 2004.

RAMALHO, J. R. Precarização do trabalho e impasses na organização coletiva no Brasil. In: ANTUNES, R. (Org.). Neoliberalismo, trabalho e sindicatos: reestruturação produtiva na Inglaterra e no Brasil. São Paulo: Boitempo, 1997. p. 85-114.

REIS, E. J. F. B. et al. Trabalho e distúrbios psíquicos em professores da rede municipal de Vitória da
Conquista, Bahia, Brasil. Cadernos de Saúde Pública, Rio de Janeiro, v. 21, n. 5, p. 1480-1490, set./out. 2005.

SANTOS, K. O. B.; ARAÚJO, T. M.; OLIVEIRA, N. F. Estrutura fatorial e consistência interna do SelfReporting Questionnaire (SRQ-20) em população urbana. Cadernos de Saúde Pública, Rio de Janeiro, v. 25, n. 1, p. 214-222, jan. 2009.

SCHNALL, P.; DOBSON, M.; ROSSKAM, E. (Org.). Unhealthy Work: causes, consequences, cures. New York: Baywood, 2009.

SOUZA, M. F. M.; SILVA, G. R. Risco de distúrbios psiquiátricos menores em área metropolitana na região Sudeste do Brasil. Revista de Saúde Pública, São Paulo, v. 32, n. 1, p. 50-58, 1998.

SOUZA, N. S. S. Perfil da população de trabalhadores do Estado da Bahia. Disponível em: $\leq$ http//www. saude.ba.gov.br/cesat/cadinfo/Artigo_IBGE.pdf $>$. Acesso em: 29 set. 2010.

STANSFELD, S. A. et al. Work characteristics predict psychiatric disorder: prospective results from the Whitehall II Study. Occupational and Environmental Medicine, v. 56, n. 5, p. 302-307, 1999.

WORLD HEALTH ORGANIZATION. User's guide to the Self-Reporting Questionnaire (SRQ). Geneva: World Health Organization, 1994. 